\title{
EFFECT OF PHASE ANGLE ON TANDEM FLAPPING-WING POWER GENERATION
}

\author{
F. KARAKAS \& I. FENERCIOGLU \\ Istanbul Technical University, Istanbul, Turkey.
}

\begin{abstract}
Two tandem wings undergoing two-dimensional sinusoidal and non-sinusoidal pitch and plunge motions are studied experimentally in a water channel at a chord-based Reynolds number of 10,000. The hindwing operates in the wake of the forewing, and its performance is affected by the vortices shed by the forewing in a tandem wing application. The vortex-wing and vortex-vortex interactions are affected by the changes in the phase angle between the fore and the hind wings. This study investigates how the changes in phase angle between the motions of the two wings play a role on the leading edge vortex (LEV) formations on the hindwing and the resulting effects on the power coefficient and the efficiency. The instantaneous lift and torque are measured by a force sensor; the velocity fields are captured by a digital particle image velocimetry (PIV) system. Sinusoidal and non-sinusoidal oscillations consisting of a pitch leading plunge motion with $\varphi=90^{\circ}$ phase angle are used for the fore and hind wing motions. Different phase angles between the fore and hindwings are tested for the tandem configuration in the range of $\psi=0^{\circ}-360^{\circ}$ with an increment of $45^{\circ}$. The pitch pivot point to point distance of two chords was set between the fore and hindwings. It is found that the phase angle between the tandem foils determines the timing and the sign of the forewing-shed LEV when the hindwing encounters this LEV. Such an interaction affects the LEV formation, growth and shedding on the hindwing and results in a change in power generation performance of the hindwing. The results further show that at this specific distance between the wings, the maximum power coefficient and efficiency occur when the phase angle between the motions of the tandem wings is near $\psi=135^{\circ}$ for the sinusoidal pitching and plunging.

Keywords: flapping wing, force measurement, PIV, power generation, tandem wings.
\end{abstract}

\section{INTRODUCTION}

Global energy demand is increasing rapidly along with the increasing population and industry. A study of World Energy Council (WEC) [1] concluded that if there will be no change in world's current way of energy use, there will be an increase of 50\%-80\% for energy demand by 2020 (Fig. 1). As the life standards are increasing and new technologies are becoming an important part of daily life, a steady and reliable supply of energy emerges as a necessity. Energy lies at the core of people's mobility and daily comfort. At present, great percentage of world's energy supply demands on fossil resources [2]. However, mankind is facing the problem of resource limitation and the disaster of environmental pollution by producing harmful gas emissions.

Accompanying with rapid increase in population, environmental problems arise and grow in a range of pollutants, hazards and ecosystem degradation over wider areas [1]. The released harmful gases catch the heat in the atmosphere and cause global warming which threaten man and the nature. To achieve efficient solutions to the environmental problems that mankind face today and generate long-term environment for sustainable development, renewable energy resources appear to be the most efficient and effective way.

This rapid growth in demand for energy has led to an increase in research on alternative and renewable energy production methods. Wind energy, hydropower, solar energy, geothermal energy and bio energies are some of the good alternatives for renewable and clean energy resources. Moreover, wind and hydropower sources have emerged as an important player in 


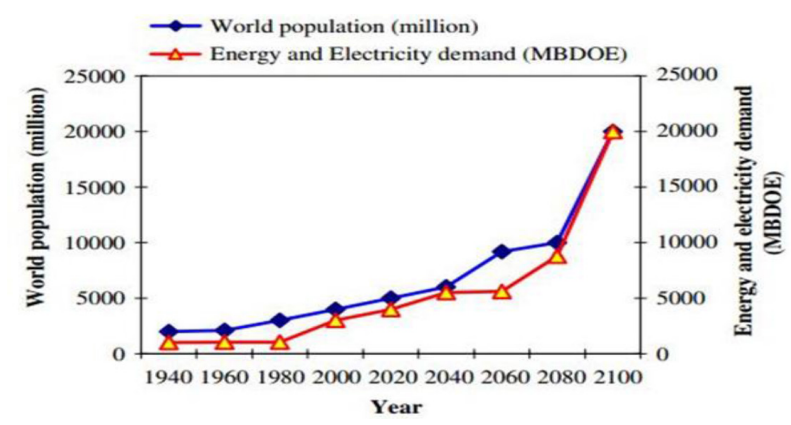

Figure 1: Annual and estimated world population and energy demand [1].

the energy space in recent decades. Especially, the comparatively large power density offered by ocean energy has made it worth to investigate new ways of extracting this energy. The average power flow intensity of $2-3 \mathrm{~kW} / \mathrm{m}^{2}$ is known in the perpendicular direction to the wave just below the ocean surface [3].

Wind and hydropower plants are widely considered as the clean and renewable sources for energy. They are also believed to be reliable and economically friendly electricity sources [4]. As research on turbine technology develops, the energy generating efficiency increases both on wind turbines and hydro turbines. However, there are also some big disadvantages of the traditional turbine-based power generation. Hydroelectric dams are notoriously expensive and have limited places to build. Wind turbine construction can be very expensive and costly to surrounding wildlife during the construction process. The noise pollution from commercial wind turbines and huge turbine blades could also give hazards to flying animals and neighboring wild lives.

In real-life applications, one of the main disadvantages regarding wind power is down to the winds' unreliability factor [5]. In many areas, the wind strength is too low to support a wind turbine or wind farm and this is where the use of flapping-wing power-generators could be great alternatives [6]. Flapping-wing power-generator could achieve similar performance levels at the low-speed environments. Moreover, reducing the viable flow speed could increase the commercially usable wind and tidal current resource bases [7].

Flapping-wing-based power generation research began in 1981 when McKinney and DeLaurier [8] proved the feasibility of a flapping-wing windmill that could generate power in a wind tunnel. Numerical parametric studies of flapping foil power production have also been conducted by Jones and Platzer [9]. They performed several numerical analyses of flapping-wing power-generator in order to verify McKinney and DeLaurier's experimental work [8] as well as attempted to find operating regimes for maximum efficiency and power extraction performance. Jones and Platzer [9] studied the transition from thrust generation to power generation, and they concluded that if the pitching amplitude is increased to a sufficiently high value, the oscillating wing could extract energy from the flowing fluid.

Most investigations on flapping-wing power-generators are based on sinusoidal motions. However, a further study by Kaya and Tuncer [10] led to the recognition of using non-sinusoidal pitching and plunging motions for higher thrust efficiency. Platzer et al. [11] defined the motion kinematics of flapping-wing power-generator by using a variable $\Delta \mathrm{T}_{R}$ where the stroke reversal time is given as a fraction of the flapping period. The variable ranges from $\Delta \mathrm{T}_{R}=0.1$ for rapid reversal to $\Delta \mathrm{T}_{R}=0.5$ for purely sinusoidal motion. Platzer et al. [12] 
and Ashraf et al. [13] numerically investigated the non-sinusoidal motion of a flapping-wing power-generator with reduced frequency of $k=0.8$ pitching amplitude of $\theta=73^{\circ}$ and plunge amplitude of $h=1.05 \mathrm{c}$. Their results showed that around $17 \%$ increase in power generation and around $15 \%$ increase in efficiency could be achieved as compared with those of sinusoidal motions. Further investigations are performed by Fenercioglu et al. [14] experimentally on flapping-wing power-generator using the same motion parameters with Platzer et al. [12] and Ashraf et al. [13], for a flapping wing with non-sinusoidal pitching and plunging motions about its quarter and mid-chord pivot points with different stroke reversal times of 0.1 (rapid motion) to 0.5 (sinusoidal motion). There is a good agreement between experimental results and 2D Navier-Stokes simulations that both show higher efficiency using non-sinusoidal motion. The Navier-Stokes computations [12,13] and experimental studies [14] confirmed the potential superiority of a non-sinusoidal oscillating power generator.

Kinsey and Dumas [15] also performed a numerical analysis on flapping-wing power generation. They proposed that the timing of the formation and shedding of the LEV during a flapping cycle is very important for achieving higher efficiency in a flapping-wing power-generator. They also found that $\varphi=90^{\circ}$ is the optimum phase angle between pitching and plunging motions for maximum energy extraction.

Zhu and Peng [16] also conducted numerical analyses on energy production by a flapping foil. They showed that the LEV can actually help the pitching of the foil and transfer energy back into the system if timed correctly. However, if timed incorrectly, increased energy is required to supply the moment from the unfavorably phased low-pressure field. With reference to an earlier study by Jones et al. [17], they pointed out that tandem configuration may increase the power output of flapping-wing power-generators.

The general summing-up from aforementioned studies is that when the pivot point is just in front of the mid-chord position and pitching motion leads plunging motion by $90^{\circ}$, the efficiency of power generation could take maximum values. So in conjunction with previous studies, the pitch pivot point is chosen as 0.44 chord lengths from the leading edge and the phase angle between pitch and plunge is chosen as $90^{\circ}$ this study, while the reduced frequency kept constant as $k=0.8$.

\section{EXPERIMENTAL SETUP}

Experiments are carried out in the close circuit, free-surface, large-scale water channel in Trisonic Laboratory at the Faculty of Aeronautics and Astronautics of Istanbul Technical University. The test section dimensions are $1010 \times 790 \mathrm{~mm}$ with turbulent intensity of under $1 \%$. Two flat plate test models with rectangular cross sections and both with chord lengths of $c=10 \mathrm{~cm}$ and spans of $s=30 \mathrm{~cm}$ are mounted in a vertical cantilevered arrangement in the water channel oscillating about their 0.44 chords between upper and lower end plates. The experimental arrangement for tandem wing configuration is shown in Fig. 2. For each test model, the connection rod connects the model to a servo motor to provide a pitching motion via a coupling system which itself is connected to a linear table which provides the plunging motion.

The particle imaging velocimetry (PIV) system is used to record the instantaneous quantitative evolution of flow structures around and in the near wake of the flat plate. The flow is illuminated by a dual cavity Nd:Yag laser (max. $120 \mathrm{~mJ} /$ pulse) oriented horizontally at the flat plate's mid-span plane. The water is seeded with silver-coated glass spheres of 10 $\mu \mathrm{m}$ mean diameter. The velocity vectors are derived from subsections of the target area of 


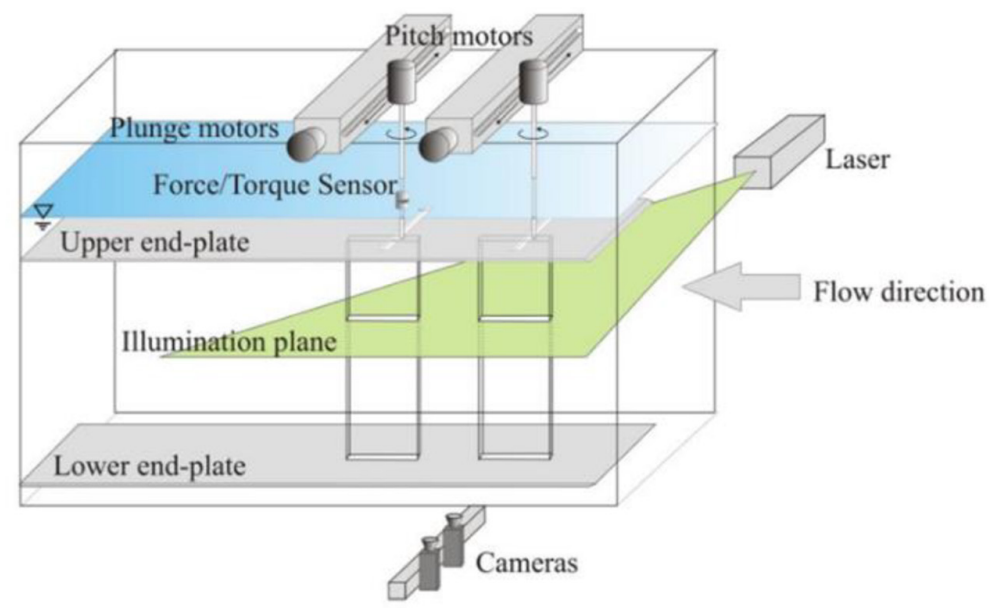

Figure 2: Experimental setup for tandem-wing power-generator.

the particle-seeded flow by measuring the movement of particles between two light pulses. Two 10-bit CCD cameras with $1600 \times 1200$ pixels resolution are positioned under the water channel to capture flow field images. The two double-frame images from the two cameras are stitched before interrogation with an in-house code using two marker points in the illumination plane for a larger field of view. The stitched images are interrogated using a double frame, cross-correlation technique with a window size of $64 \times 64$ pixels and $50 \%$ overlapping in each direction. During the experiments, 32 images were acquired for one period of the flat plate for a total of three periods of oscillatory motion.

A six-component Force/Torque (F/T) sensor, which is be able to work in water and meantime measure, is used to measure the forces and moments acting on the flapping foil. The sensor range and resolution are $\pm 50 \mathrm{~N}$ for force in $x$ and $y$ directions and $\pm 500 \mathrm{Nmm}$ for torque. Measurement uncertainty with $95 \%$ confidential level in percent of full scale load is $1.50 \%$ for $F_{x}$ and $F_{y}$ and $2.00 \%$ for $T_{z}$. The measurement uncertainty values are the maximum amount of error expressed as a percentage of its full-scale load. The sensor is attached to the vertical cantilevered arrangement between the flat plate model and the pitch servo motor, oriented with its cylindrical $z$-axis normal to the pitch-plunge plane. The $x y$-plane of the sensor is mounted to the airfoil root which is parallel to the lift and drag forces plane. The pitching moment also coincide with the $z$-axis.

In order to eliminate the force and moment effects because of the moment of inertia of the motors, experiments are repeated in air with the same conditions. The aerodynamic force and moment data in air is subtracted from the original data in water. In order to eliminate the bias error in the measurements reference data is taken before and after each case of experiment. Dynamic force and moment data are collected for 30 periods with a sampling rate of 10,000 $\mathrm{Hz}$ for all experiments conducted in the scope of this study. The data was block averaged within a window of 100 data points which resulted in an effective $100 \mathrm{~Hz}$ sampling rate and then phase averaged to downscale data to one period. A parabolic low-pass FFT filter was applied to reduce the high-frequency noise and to omit the effect of natural frequency of the system.

The kinematic motions of the foil are non-sinusoidal pitching and plunging where the plunging motion is followed by rapid pitching reversals. The motion of the single foil is given 

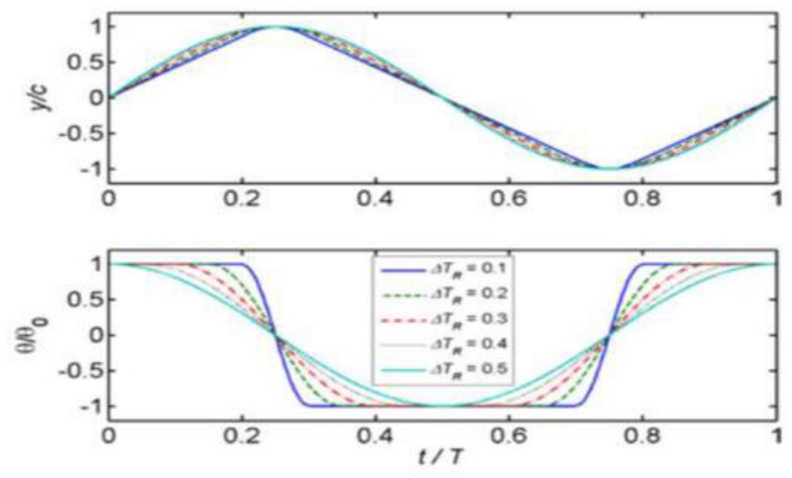

Figure 3: Variation of plunge and pitch motions [10].

in Fig. 3, with a period of constant translational velocity combined with a constant pitch angle, followed by a sinusoidal reversal of direction and pitch angle. The motion is shown with varying reversal times as a fraction of the total cycle, $\Delta \mathrm{T}_{R}$, ranging from $\Delta \mathrm{T}_{R}=0.1$ for rapid reversal to $\Delta \mathrm{T}_{R}=0.5$ for purely sinusoidal motion.

The non-dimensional plunge motion amplitude $h$ is 1.05 and the pitch motion amplitude $\theta_{o}$ is $73^{\circ}$ as consistent with previous studies $[13,14]$. The reduced frequency is set constant as $k=0.8$ throughout this study. The Reynolds number considered for this study is $\operatorname{Re}=10,000$ with the corresponding flapping frequency of $f=0.125 \mathrm{~Hz}$ and free stream velocity of $U_{\infty}=$ $0.1 \mathrm{~m} / \mathrm{s}$ in the experiments. The non-dimensional distance between tandem foil pivot points was set to $L_{x}=2 c$ and $\psi$ defines the phase angle between the motions of the tandem wings, by which the trailing wing leads the fore wing.

\section{RESULTS AND DISCUSSIONS}

In this section, the power generated from close configuration of two flapping foils in tandem is studied. For each individual foil, sinusoidal $\left(\Delta \mathrm{T}_{R}=0.5\right)$ and non-sinusoidal $\left(\Delta \mathrm{T}_{R}=0.3\right.$ and 0.4 ) motions with pitch leading plunge by $\varphi=90^{\circ}$ phase angle are tested. To understand the performance of the tandem foil generator, the power coefficient in hind wing is measured using a force sensor. The efficiency of power extraction is defined as the ratio of the power extracted by hind foil divided by the power available for the system. The phase angle between the leading and the trailing foils are examined between $\psi=0^{\circ}$ to $360^{\circ}$ with increments of $45^{\circ}$.

Figure 4 shows the vorticity patterns for half period of the oscillating cycle of the tandem foils both with sinusoidal flapping. The flow is from left to right in the figure and the first image shows the flat plates at their start-up position $(t=0)$. Since the phase angle between the foils is set to $\psi=270^{\circ}$ where the trailing wing leads the fore wing, the hind wing is located at its minimum plunge position while the fore wing is at mid-plunge position. Both wings move upward and the maximum plunge amplitude position of the fore wing corresponds to the image at $t=8 T / 32$ which is followed by a sinusoidal pitch reversal $\left(\Delta \mathrm{T}_{R}=0.5\right)$. The fore wing continues its downward motion after reversal of direction and pitch angle while the hind wing still continues its upward motion. The last image for each case at $t=15 T / 32$ shows the fore wing approaching its mid-plunge position during downstroke and the hind wing approaching its maximum plunge position.

At $\psi=270^{\circ}$ phase angle, high lift is generated on the hind foil during the stroke reversal because of the interaction with the vortex wake of the leading foil, but the plunge velocity of 


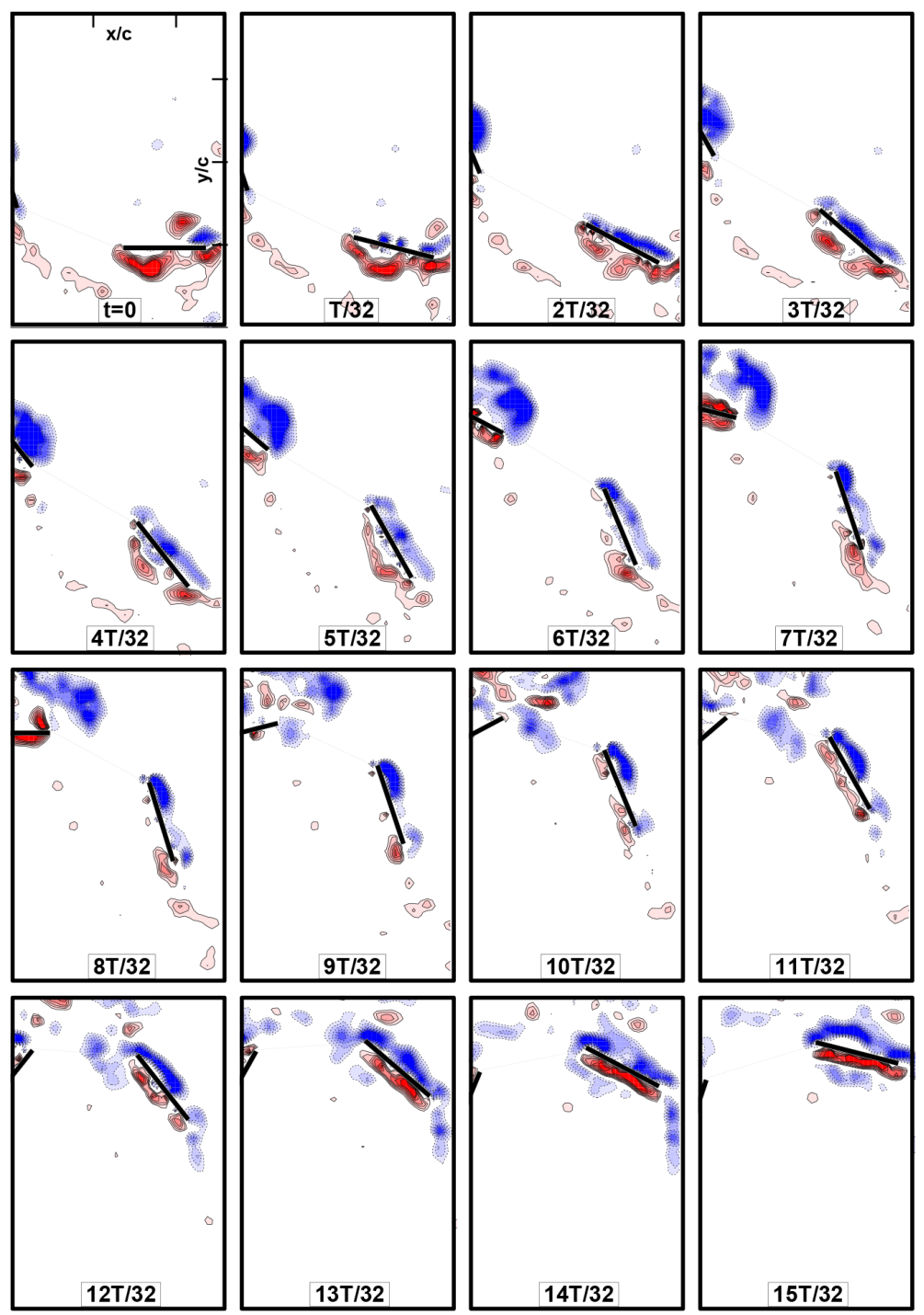

Figure 4: Vorticity patterns for half period of the motion $\left(\Delta \mathrm{TR}=0.5, \psi=270^{\circ}\right)$.

the hind foil has opposite sign to the lift as can be seen Fig. 5a where the instantaneous lift coefficient plots are given. Therefore, a positive power output could not be achieved at this phase angle. In comparison at $\psi=135^{\circ}$, as shown in Fig. 5b, the phase difference between the foils provide better synchronization of lift with the plunge velocity, resulting in net positive power output being maintained throughout the whole cycle. The details of the negative interaction between the vortex wake of the leading foil with the trailing foil due to the effect of $\psi$ is illustrated in the vorticity patterns in Fig. 6 where maximum mean power coefficient is achieved. The poor synchronization of lift with the hind foil's plunge velocity causes the net power to drop down to below zero as can also be seen in Fig. 7.

Phase angle $\psi=135^{\circ}$ appears to be the better choice of parameter value for the sinusoidal tandem-wing power generation system. This phase angle was also suggested as the best 


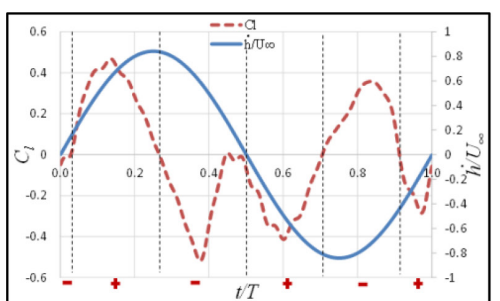

(a)

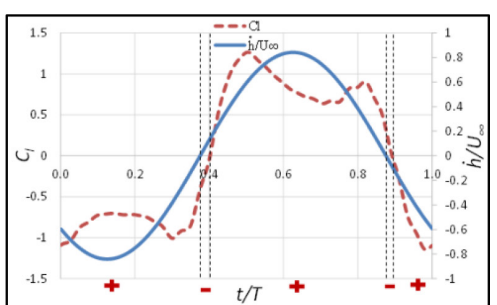

(b)

Figure 5: Instantaneous $\mathrm{Cl}$ variation for $\Delta \mathrm{TR}=0.5$ with (a) $\psi=270^{\circ}$ (b) $\psi=135^{\circ}$.

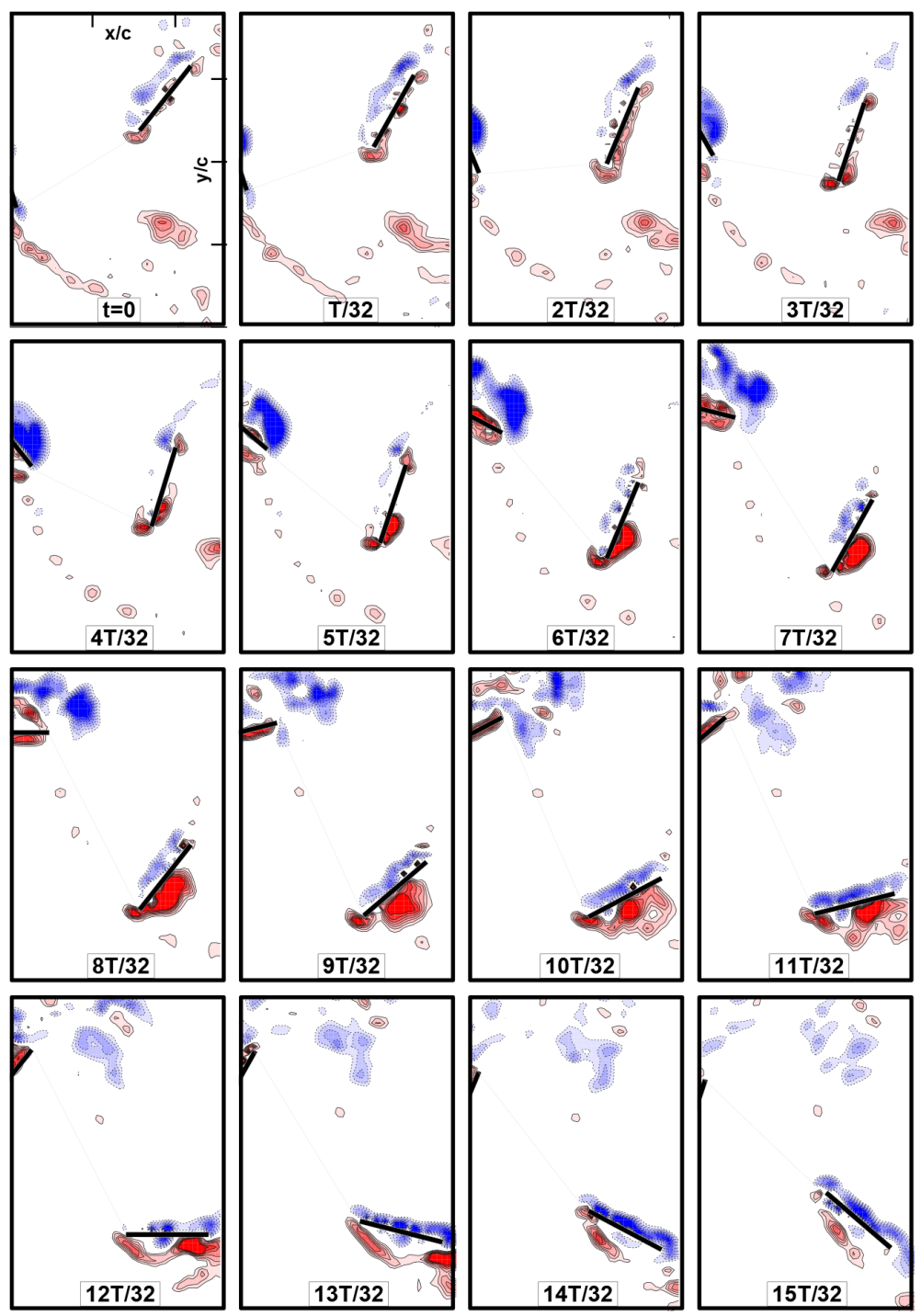

Figure 6: Vorticity patterns for half period of the motion $\left(\Delta \mathrm{TR}=0.5, \psi=135^{\circ}\right)$. 


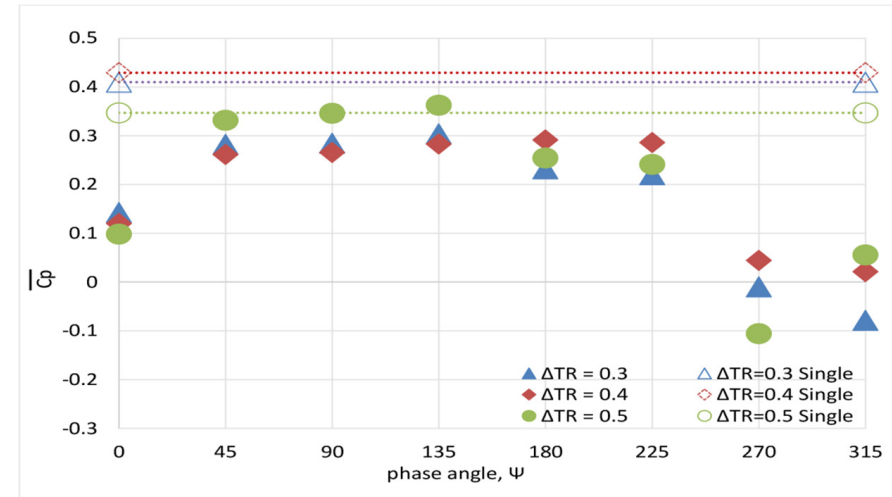

Figure 7: Mean power coefficient variations with phase angle for the hindwing.

power generation choice by Ashraf [18] in his numerical study, showing the good agreement between the experiment and numerical solutions.

At the instants of $t=12 T / 32$ and $t=26 T / 32$, the wake of the leading foil strikes the leading edge of the trailing foil when the phase angle is set to $\psi=270^{\circ}$, as can be seen in Fig. 4, creating a large lift in the opposite direction to the motion of the trailing foil which can be observed in Fig. 5a. However, at these same instants, the same vortices are avoided by the trailing foil in phase angle $\psi=135^{\circ}$ as shown in Fig. 6, resulting in less reduction in the net power. The wake of the trailing foil avoids the interaction with the main vortex shedding of the leading foil due to a better placement of the foil in its wake.

The hindwing results are compared to a single wing to determine the effect of the forewing on the hindwing in Fig. 7. The power coefficient for the hindwing varies much more drastically from the single wing results for different phase angles. This is very likely due to the change in vortex interactions between the shed vortex from the forewing and the LEV of hindwing, caused by different phase angles. For the sinusoidal case when the tandem wings flap with $\psi=0^{\circ}$ phase lag, the hindwing experiences large increases in the peak lift. However, with increasing phase lag, the peak lift coefficient decreases for the hindwing. Moreover, for $\psi=180^{\circ}$ and $225^{\circ}$, the peak lift coefficient is even lower than that of the single-wing case. There is also poor synchronization in the lift and plunge velocity of the hind wing at $\psi=$ $270^{\circ}$, which is the only case that gives negative mean power coefficient for the sinusoidal cases. Notably, this unfavorable synchronization causes the hindwing of the $\psi=270^{\circ}$ case to produce positive lift at the downstroke and negative lift at the upstroke, whereas the case with the highest mean power coefficient of the $\psi=135^{\circ}$ produces most of its positive lift during the upstroke and negative lift during the downstroke. Figure 7 shows that the power and efficiency results are sensitive to $\psi$, indicating that the system might needs to be controlled by varying $\psi$ to achieve the optimal positioning of the trailing foil in the vortex wake of the leading foil. For the sinusoidal case when the phase angle is $\psi=135^{\circ}$, hindwing outperforms the single wing and generates $4.57 \%$ higher mean power.

In Fig. 8, the trailing foil avoids the shed vortex generated by the leading foil throughout its whole period of motion due to the $\psi=90^{\circ}$ phase lag with the leading foil. The vorticity contours show similar positioning as compared with a previous study also conducted using the same experimental setup where only $\psi=0^{\circ}, 90^{\circ}$ and $180^{\circ}$ were investigated [19]. Parallel to the sinusoidal tandem power generation, the non-sinusoidal motion results are also sensi- 


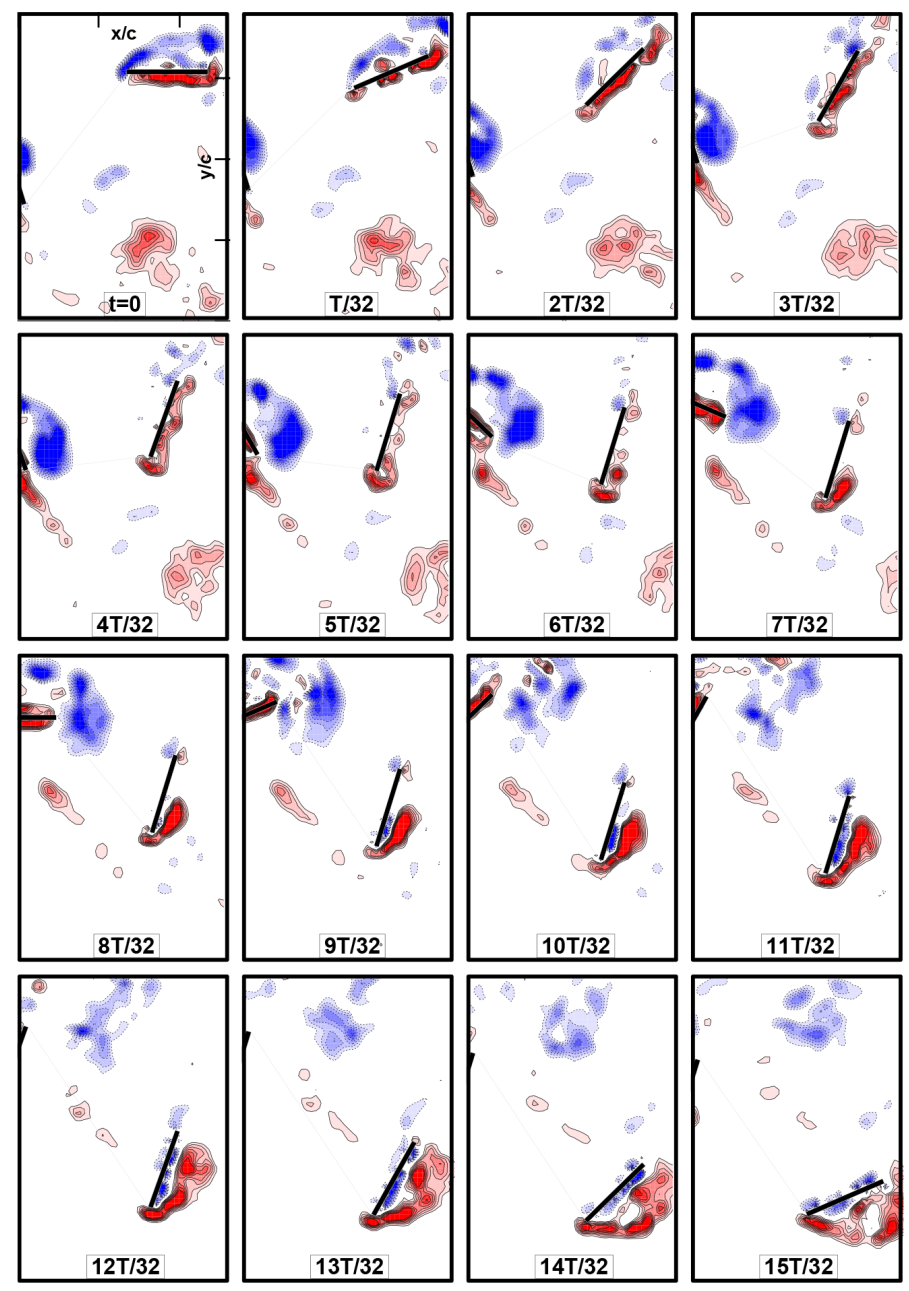

Figure 8: Vorticity patterns for half period of the motion $\left(\Delta \mathrm{TR}=0.3, \psi=90^{\circ}\right)$.

tive to phase angle $\psi$ and again indicating that the trailing foil needs to be placed optimally in the vortex wake of the leading foil. Compared with a single wing, the placement of hindwing results in very different power generation performance. The hindwing shows a decrease in the mean power coefficient regardless of the phase angle for the non-sinusoidal cases of $\Delta \mathrm{T}_{R}$ $=0.3$ and 0.4 .

The size of the LEV generated corresponds to the peak lift production. The timing of the LEV generation and shedding corresponds to the phase lag observed in the lift data. This suggests that changes in the phase angle can be used to control the force production and efficiency of the hindwing by controlling its LEV formation. If the directions of rotation of the shed vortex and LEV are opposite to each other, there is a destructive vortex interaction. This suppresses the LEV formation and quickens the shedding resulting in the formation of a smaller LEV compared with the case of single wing without interaction. 


\section{CONCLUSIONS}

In this experimental study, effects of phase angle between wings in tandem arrangement on power generation are examined. The flow structures and the forces acting on flat plates performing sinusoidal and non-sinusoidal pitching and plunging motions are investigated via detailed quantitative flow visualization and simultaneous direct force measurements. Reynolds number of 10,000, non-dimensional plunge amplitude of $h / c=1.05$, pitch amplitude of $\vartheta_{\mathrm{o}}=73^{\circ}$ and phase angles of $\phi=90^{\circ}$ between pitch and plunge are considered in all experiments. The pitch pivot for both wings was positioned at 0.44 chord location from their leading edges.

The power extraction and efficiency of the hindwing are greatly affected by its interaction with the wake of the forewing. The interaction process can be controlled by adjusting the phase angle between the two wings. The LEV generation of the hindwing is greatly affected by the interaction between the shed vortex of the forewing and the hindwing. Phase angle changes the timing of the interaction and determines the direction of rotation of the vortex that meets the LEV of hindwing. This interaction reinforces the LEV and results in larger and stronger LEV formation compared with the case of single wing with no interaction. Thus, the timing of the generation, strength and shedding of the LEV on the hindwing are adjusted to maximize the power generation. The interaction between the shed vortex and the LEV can be described as constructive or destructive. Most of the studied cases exhibit constructive vortex interaction, where the directions of rotation of the shed vortex and the LEV are the same. The hindwing outperforms the single wing by $4.57 \%$ for the sinusoidal case when the phase angle is $\psi=135^{\circ}$. However, if positioned incorrectly, as $\psi=270^{\circ}$, power could not be extracted.

\section{ACKNOWLEDGEMENT}

This study is funded by the Scientific and Technological Research Council of Turkey (TUBITAK) Grant 214M385.

\section{REFERENCES}

[1] Omer, A., Energy, environment and sustainable development. Renewable and Sustainable Energy Reviews, 12(9), pp. 2265-2300, 2008. http://dx.doi.org/10.1016/j.rser.2007.05.001

[2] Chu, S. \& Majumdar, A., Opportunities and challenges for a sustainable energy future. Nature, 488, pp. 294-303, 2012.

http://dx.doi.org/10.1038/nature11475

[3] Falnes, J., A review of wave-energy extraction. Marine Structures, 20(4), pp. 185-201, 2007.

http://dx.doi.org/10.1016/j.marstruc.2007.09.001

[4] Schiermeier, Q., Tollefson, J., Scully, T., Witze, A. \& Morton, O., Electricity without carbon. Nature, 454, pp. 816-823, 2008.

http://dx.doi.org/10.1038/454816a

[5] Ragheb, M. \& Ragheb, A., Wind Turbines Theory-the Betz, Equation and Optimal Rotor Tip Speed Ratio, INTECH Open Access Publisher, 2011. http://dx.doi.org/10.5772/21398

[6] Young, J., Lai, J.C.S. \& Platzer, M.F., A review of progress and challenges in flapping foil power generation. Progress in Aerospace Science, 67, pp. 2-28, 2014.

http://dx.doi.org/10.1016/j.paerosci.2013.11.001

[7] Pearce, F., Current power: new tide turbines tap oceans of energy. New Scientist, 2830, pp. 48-51, 2011. 
[8] McKinney, W. \& DeLaurier, J., Wingmill: an oscillating-wing windmill. Journal of Energy, 5(2), pp. 109-115, 1981. http://dx.doi.org/10.2514/3.62510

[9] Jones, K.D. \& Platzer, M.F., Numerical computation of flapping wing propulsion and power extraction. 1997.

[10] Kaya, M. \& Tuncer, I., Nonsinusoidal path optimization of a flapping airfoil. AIAA Journal, 45(8), 2007.

http://dx.doi.org/10.2514/1.29478

[11] Platzer, M.F., Ashraf, M.A., Young, J. \& Lecturer, S., Wind and hydropower generator. New Horizons, pp. 1-13, 2009.

[12] Platzer, M., Ashraf, M., Young, J. \& Lai, J., Extracting power in jet streams: pushing the performance of flapping-wing technology. 27th International Congress of the Aeronautical Sciences, 2010.

[13] Ashraf, M.A., Young, J., Lai, J.C.S. \& Platzer, M.F., Numerical analysis of an oscillating-wing wind and hydropower generator. AIAA Journal, 49(7), pp. 1374-1386, 2011. http://dx.doi.org/10.2514/1.J050577

[14] Fenercioglu, I., Zaloglu, B., Young, J., Ashraf, M.A., Lai, J.C.S. \& Platzer, M.F., Flow structures around an oscillating wing power generator. AIAA Journal, 53(11), pp. 33163326, 2015. http://dx.doi.org/10.2514/1.J053950

[15] Kinsey, T. \& Dumas, G., Parametric study of an oscillating airfoil in a power-extraction regime. AIAA Journal, 46(6), pp. 1318-1330, 2008. http://dx.doi.org/10.2514/1.26253

[16] Zhu, Q. \& Peng, Z., Mode coupling and flow energy harvesting by a flapping foil. Physics Fluids, 21(3), 2009. http://dx.doi.org/10.1063/1.3092484

[17] Jones, K.D., Lindsey, K. \& Platzer, M., An investigation of the fluid-structure interaction in an oscillating-wing micro-hydropower generator. Advance in Fluid Mechanics, 36, pp. 73-84, 2003.

[18] Ashraf, M.A., Numerical simulation of the flow over flapping airfoils in propulsion and power extraction regimes. University of New South Wales, Australian Defence Force Academy, School of Engineering and Information Technology, 2010.

[19] Fenercioglu, I., Zaloglu, B., Ashraf, M., Young, J., Lai, J. \& Platzer, M.F., Flow around an oscillating tandem-wing power generator. In 53rd AIAA Aerospace Sciences Meeting, p. 1751, 2015.

http://dx.doi.org/10.2514/6.2015-1751 